\title{
EVALUATION OF CAUSATIVE FACTORS IN AMLODIPINE INDUCED PEDAL EDEMA
}

\author{
KIRAN SHETTY1 ${ }^{1}$ RANJAN SHETTY K. ${ }^{*}{ }^{*}$, PRAGNA RAO ${ }^{2}$, VIVEK G. ${ }^{3}$, NAVEENCHANDRA G. S. ${ }^{4}$, ABDUL RAZAK ${ }^{1}$, \\ DEEPAK $^{1}$, AMRUTH KIRAN $^{5}$
}

${ }^{1}$ Department of Cardiology, Kasturba Medical College, Manipal University, Manipal, India, ${ }^{2}$ Department of Biochemistry, Kasturba Medical College, Manipal University, Manipal, India, ${ }^{3}$ Consultant Cardiologist, BGS Global Hospital, Bengaluru, India, ${ }^{4}$ Consultant Cardiologist, Columbia Asia Hospital, Bengaluru, India, ${ }^{5}$ Department of Pharmacology, MMMC, Manipal University, Manipal, India

Email: ranjanshettyk@yahoo.com

Received: 11 Aug 2016 Revised and Accepted: 21 Sep 2016

\begin{abstract}
Objective: To study the edema causing factors in hypertensive, amlodipine-induced pedal edema patients.

Methods: The present was a prospective, observational study. A total of one hundred and twenty-four essential hypertensive patients, of either gender attending the outpatient department of cardiology and medicine, were recruited for this study. Out of the 124 patients, 62 were of the amlodipine-induced pedal edema [AIPE] group and other 62 patients were amlodipine-treated non-edema [ATNE] group. All the patients were receiving a dosage of amlodipine $5 \mathrm{mg} /$ day. All recruited patients completed the study. The present study conducted at Kasturba Hospital, Manipal

Results: The vanillyl mandelic acid (VMA) (mean \pm SD) $7.08 \pm 2.3 \mathrm{mg} / 24 \mathrm{~h}$ and $4.9 \pm 1.7 \mathrm{mg} / 24 \mathrm{~h}$ in AIPE and ATNE groups respectively. Blood pressure (BP) and VMA was higher in AIPE group than the ATNE group ( $<<0.001)$. Pulse rate (PR), serum proteins, creatinine, sodium, osmolality,
\end{abstract} did not show any significant difference between the two study groups.

Conclusion: In essential hypertensive patients with AIPE group presented with a higher VMA level than the ATNE group. The elevated catecholamine's possibly the causative factor for AIPE.

Keywords: Calcium channel blocker, Amlodipine, Pedal edema, Causative factors

(C) 2016 The Authors. Published by Innovare Academic Sciences Pvt Ltd. This is an open access article under the CC BY license (http://creativecommons.org/licenses/by/4. 0/) DOI: http://dx.doi.org/10.22159/ijpps.2016v8i11.14650

\section{INTRODUCTION}

Hypertension (HTN) is a major global health impact. The global burden of HTN is extremely high and globally one billion people are affected by HTN and in India; it has reached to $29.8 \%$ HTN is most common risk factors for cardiovascular, cerebrovascular and renal diseases [1, 2]. In most instances, it is asymptomatic, until and unless it affects the major organs like heart, kidney, cerebrovascular, peripheral vascular system including the eye. Early detection and treatment will reduce the risk and improves patient's quality of life [3-5].

According to Joint National Committee eighth report (JNC-8), in a general population, the antihypertensive drug therapy should be started when BP is $\geq 150 / 90 \mathrm{~mm}$ Hg in adult's $\geq 60 \mathrm{y}$. In patients $\leq 60$ $\mathrm{y}$, initiate the therapy and targeted goals should be $\leq 140 / 90 \mathrm{~mm} \mathrm{Hg}$, the same recommendation remains for the patient's age $\geq 18 \mathrm{y}$ with either chronic kidney disease (CKD) or diabetes. The initial antihypertensive treatment regimen should be a thiazide diuretic, Calcium channel blockers (CCBs), angiotensin converting enzyme inhibitors (ACEI) or angiotensin receptor blockers (ARBs), in the white population; initial therapy should be a thiazide-type diuretic or CCBs in a black population [6].

BP can be controlled by lifestyle modifications or with antihypertensive agents or both. The current pharmacological options to treat HTN include diuretics, CCBs, ACEIs, ARBs, sympatholytic drugs and vasodilators. The choice of drug therapy will be determined by the severity of diseases and associated patient factors $[1,7]$.

CCBs acts on voltage-dependent $\mathrm{Ca}++$ channels. The subtype includes L-type, N-type, T-type, P/Q-type and N-type, and they are widely spread throughout the body and play a crucial role in the maintenance of vascular tone [8]. Amlodipine is a third generation dihydropyridine group of CCB. Amlodipine is a very potent and long acting drug and also a well-tolerated antihypertensive agent. Ankle edema is one of the most common and frequent adverse effects of amlodipine. Peripheral edema is uncomfortable, sometimes intolerable and may lead to a decline in patient's quality of life [9, 10]. The incidence of pedal edema ranges from $1.7 \%$ to $63.3 \%$ [7]. Monotherapy showed a higher incidence of pedal edema than the combination therapy [8]. According to literature, about $9.3 \%$ of patients discontinue therapy because of its adverse effects. Overcoming this important issue is to stop amlodipine therapy and switch to another group of antihypertensives. Usually, diuretics will not be effective in the resolution of CCBs induced pedal edema [11].

Edema is a condition where free fluids accumulate in the interstitial space. The important mechanism involves, the increased capillary hydrostatic pressure, decreased plasma oncotic pressure; increased capillary permeability and obstruction of the lymphatic system. However, there are many mechanisms postulated for AIPE, the exact mechanism and causative factors of ankle edema by CCBs is not clearly understood $[11,12]$.

The purpose of this study was to evaluate the causative factors in amlodipine-induced pedal edema and to compare the clinical parameters and biochemical parameters in hypertensive AIPE and ATNE groups.

\section{MATERIALS AND METHODS}

\section{Methods}

The present was a prospective, observational study. A total of one hundred and twenty-four hypertensive patients of either gender attending the outpatient department of cardiology and medicine recruited for this study. The patient's information sheet was given to all patients and explained about the present study; consent received before the study commences. Out of the 124 patients, 62 were of AIPE group and other 62 patients were in ATNE group. All the patients were receiving a dosage of amlodipine $5 \mathrm{mg} /$ day. All recruited patients completed the study. The study protocol was confirmed, and approval of the Institutional Ethics Committee (Approval no IEC 681/2013). The present study conducted at Kasturba Hospital, Manipal, Karnataka, India. 


\section{Inclusion criteria}

Hypertensive patients of either gender [ $>140 / 90 \mathrm{~mm} \mathrm{Hg}$, currently receiving amlodipine more than six months as an antihypertensive therapy. The age limit was 18 to $70 \mathrm{y}$. For AIPE group patients should develop AIPE [with no other obvious cause], and for ATNE group all the inclusion criteria were same except, patients should not develop edema.

\section{Exclusion criteria}

We excluded the patients for following reasons, patients with major organ failure, endocrine abnormalities like Cushing's syndrome, hypothyroidism. Pregnant women and women on oral contraceptive pills, patients on other class of antihypertensive agents, nonsteroidal anti-inflammatory drugs, and steroids, patients with lymphedema, pulmonary hypertension, secondary hypertension, patients with the varicose vein, venous insufficiency. Exclusion criteria are same for both the study groups.

\section{Study procedure}

In the present study, a total of 124 patients [n $=124]$ recruited who met the inclusion criteria. The consultant cardiologist examined the patients. The BP measured using standard mercury sphygmomanometer. Three consecutive readings of BP and PR noted at an interval of 10 min. Pedal edema was confirmed by clinical method over the medial malleolus of both legs. After initial screening, demographic parameters, family history, clinical examination findings and biochemical parameters were also noted. All 124 patients who are on amlodipine $5 \mathrm{mg}$ /day as an antihypertensive treatment and in that 62 patients are in AIPE group, and other 62 patients are in ATNE group.

\section{Statistical analysis}

Descriptive statistics (mean $\pm \mathrm{SD}$, Median, IQR, and Percentage) provided for demographic variables. Categorical variables were compared using Chi-square test, and continuous variables compared using independent t-test. The Mann-Whitney test compared the skew variables. All the analysed by SPSS v 15, p<0.05, was considered to be statistically significant.

\section{RESULTS}

It was a prospective, observational study. Hence, all 124 recruited patients completed the study. The patient demographic parameters compared in table no 1, the patient's age was found to be $57.4 \pm 10.3$ and $58.5 \pm 8.5 \mathrm{y}$ in AIPE and ATNE groups respectively. Weights of AIPE and ATNE groups were $64.9 \pm 10.7$ and $61.85 \pm 9.3 \mathrm{kgs}$. respectively. The mean height of patients in AIPE and ATNE groups was $157.3 \pm 10.3$ and $159.3 \pm 9.1 \mathrm{cms}$. respectively. BMI was $25 \pm 2.7$ in both the groups. Type 2 Diabetic Mellitus (DM) in AIPE group was 24 [38.7\%], and ATNE group was 26 [41.9\%]. Demographic parameters and all other aspects, compared between the groups, there was no significant difference between them.

Table 1: Comparison of demographic parameters of AIPE and ATNE groups

\begin{tabular}{lllll}
\hline S. No. & Variables & AIPE [n=62] [mean \pm SD] & ATNE [n=62] [mean \pm SD] & P-VALUE* \\
\hline 1 & Age[years] & $57.4 \pm 10.3$ & $58.5 \pm 8.5$ & 0.53 \\
2 & Weight [Kgs] & $61.85 \pm 9.3$ & $64.9 \pm 10.7$ & 0.28 \\
3 & Height [Cm] & $157.3 \pm 10.3$ & $159.3 \pm 9.1$ & 0.25 \\
4 & BMI & $25 \pm 2.7$ & $25 \pm 2.7$ & 0.34 \\
5 & T2 DM & $24[38.7 \%]$ & $26[41.9 \%]$ & 0.86 \\
6 & Gender [M/F] & $31 / 31[50 \% / 50 \%]$ & $38 / 24[61.3 \% / 38.7 \%]$ & 0.19 \\
\hline
\end{tabular}

The values are expressed in mean \pm SD (\%). Categorical variables were compared using Chi-square test; continuous variables are compared by independent $\mathrm{t}$-test. ${ }^{*} \mathrm{P}<0.05$, was consider to be statistically significant.

\section{Abbreviation}

AIPE: Amlodipine induced pedal edema, ATNE: Amlodipine treated non edema BMI: Body mass index, DM: Diabetic mellitus, Kgs: Kilograms, Cm: Centimeters, M/F: Male/Female.
Table 2, Comparison of the clinical parameters between AIPE and ATNE groups. The systolic and diastolic blood pressure (SBP and DBP) showed a significant increase in AIPE group than the ATNE group. PR and LV ejection Fraction did not show any significant difference between the two study groups.

Table 2: Comparison of clinical parameters between AIPE and ATNE groups

\begin{tabular}{llll}
\hline S. No. & Variables & AIPE [n=62] [mean \pm SD] & ATNE [n=62] [mean \pm SD] \\
\hline 1 & SBP [mmHg] & $142.8 \pm 3.6$ & $138.9 \pm 4.4$ \\
2 & DBP [mmHg] & $83.65 \pm 4.6$ & $80.24 \pm 4.4$ \\
3 & PR [B/M] & $78 \pm 9$ & $77 \pm 8$ \\
4 & LV F [\%] & $65 \pm 5$ & $65 \pm 3$ \\
5 & IVC [mm] & $15 \pm 1.5$ & $15 \pm 1.5$ \\
\hline
\end{tabular}

The values expressed in mean $\pm \mathrm{SD}$. Continuous variables compared by independent $\mathrm{t}$-test. ${ }^{*} \mathrm{P}<0.05$. Is consider being statistically significant

\section{Abbreviation}

AIPE: Amlodipine induced pedal edema, ATNE: Amlodipine treated no edema, SBP: Systolic blood pressure, DBP: Diastolic blood pressure, PR: Pulse rate, LVF: Left ventricular ejection fraction, IVC: Inferior vena cava, B/M: Beats/minutes, mm: Millimeters.

Table 3, Comparison of the biochemical parameters between AIPE and ATNE groups. VMA (mean \pm SD) $7.08 \pm 2.3 \mathrm{mg} / 24 \mathrm{~h}$ and $4.9 \pm 1.7 \mathrm{mg} / 24 \mathrm{~h}$ in AIPE and ATNE groups respectively. AIPE group showed increased VMA of $2.18 \mathrm{mg} / 24 \mathrm{~h}$, which is statistically significant. We put ROC curve for VMA, which covers $76.8 \%$ Area under the curve (AUC) with specificity $72.6 \%$ and sensitivity $71.2 \%$. The cut-off value was $5.65 \mathrm{mg} / 24 \mathrm{~h}$.

More than the VMA cutoff value patients have $6.4 \%$ higher chance to cause of AIPE. (Odds ratio was 6.4). We compared the other parameters are like total serum protein, albumin, globulin, creatinine, sodium, and osmolality, between the two groups. There was no significant difference seen between the two groups. 
Table 3: Comparison of clinical parameters between AIPE and ATNE groups

\begin{tabular}{|c|c|c|c|c|}
\hline S. No. & Variables & AIPE [n=62] [mean \pm SD] & ATNE [n=62] [mean \pm SD] & P-Value* \\
\hline 1. & Serum creatinine $[\mathrm{mg} / \mathrm{dL}]$ & $1 \pm 0.23$ & $1 \pm 0.21$ & 0.745 \\
\hline 2. & Total protein $[\mathrm{g} / \mathrm{dL}]$ & $7.5 \pm 0.44$ & $7.5 \pm 0.49$ & 0.123 \\
\hline 3. & Serum albumin $[\mathrm{g} / \mathrm{dL}]$ & $4.5 \pm 0.46$ & $4.3 \pm 0.38$ & 0.049 \\
\hline 4. & Serum globulin $[\mathrm{g} / \mathrm{dL}]$ & $3.07 \pm 0.37$ & $3.1 \pm 0.58$ & 0.391 \\
\hline 5. & Serum Na+[mmol/l $]$ & $139.5 \pm 3.21$ & $138.8 \pm 3.38$ & 0.233 \\
\hline 6. & Serum osmolality [mosml/kg] & $288.3 \pm 12.51$ & $289.1 \pm 9.7$ & 0.708 \\
\hline 7. & VMA [mg/24 h] & $7.08 \pm 2.3$ & $4.9 \pm 1.7$ & $<0.001$ \\
\hline
\end{tabular}

The values expressed in mean \pm SD. Continuous variables compared by independent t-test. $*$ P $<0.05$ considered to be statistically significant

\section{Abbreviation}

AIPE: Amlodipine induced pedal edema, ATNE: Amlodipine treated no edema, VMA: Vanillyl mandelic acid

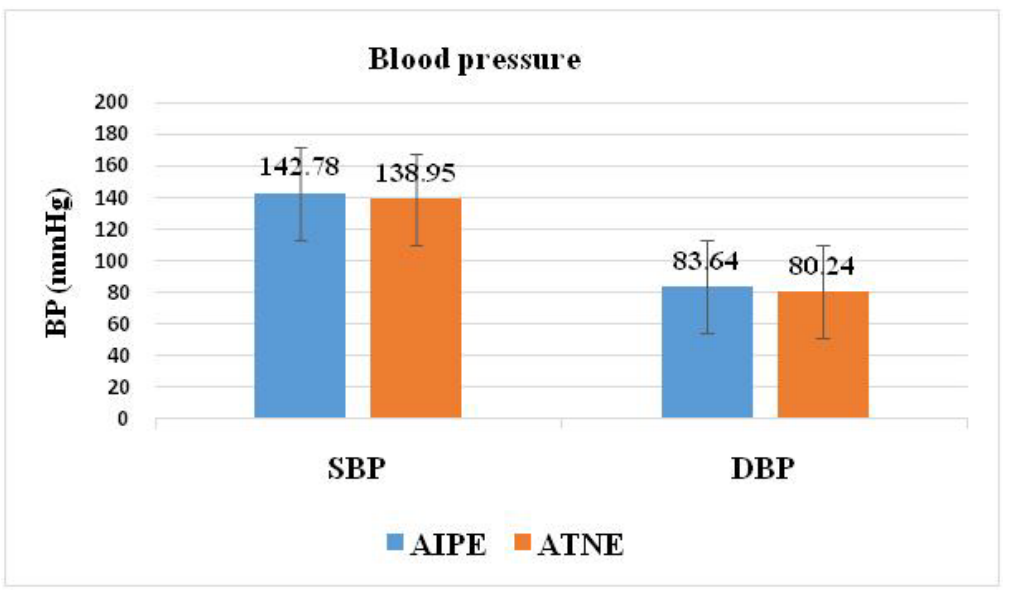

Fig. 1: Error diagram showing the comparison of SBPand DBP between AIPE and ATNE group ( $n=62$ in each group). AIPE group showed significantly higher SBPandDBP than ATNE group $(\mathrm{P}<0.05)$

\section{Abbreviation}

AIPE: Amlodipine induced pedal edema, ATNE: Amlodipine treated no edema, SBP: Systolic blood pressure, DBP: Diastolic blood pressure

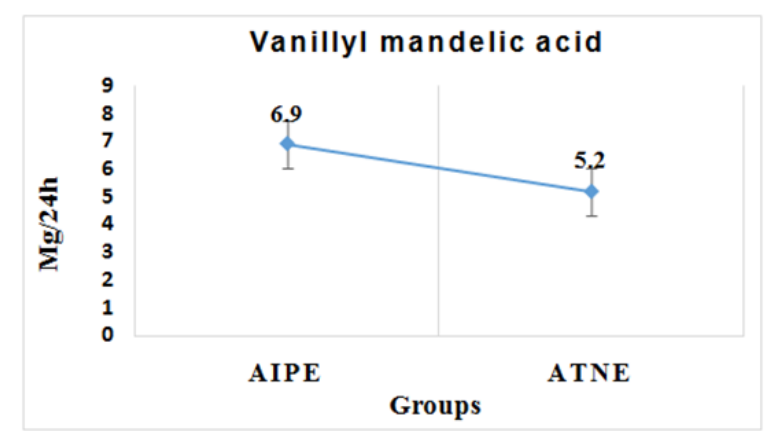

Fig. 2: Line diagram showing a comparison of vanillyl mandelic acid (VMA), between Amlodipine induced pedal edema group (AIPE) and amlodipine-treated non edema group(ATNE) ( $n=62$ in each group). AIPE group showed significantly higher VMA level than the ATNE group $(P<0.05)$

\section{DISCUSSION}

Amlodipine is a third generation [13], dihydropyridine group of CCB, it is commonly used an antihypertensive agent, because of its excellent pharmacological properties. The main drawback of this drug is which produces of pedal edema in a wide range. AIPE patients show decreased the quality of life regarding self-care, mobility, pain, and discomfort. Edema causes more anxiety in hypertensive patients [14]. People who are suffering from anxiety were two to three times more likely to develop hypertension [15]. In our study, the BP was significantly higher in AIPE group than the
ATNE group, even though they are on same antihypertensive medication. Many studies have explained the direct relationship between hypertension and anxiety [16]. Pedal edema which causes psychosocial stress which associated with anxiety and which stimulates the autonomic nerves system (ANS), which leads to increase in circulating catecholamines [17]. There are many mechanisms postulated for AIPE, and the principal mechanism involves interference of normal autoregulatory postural vasoconstrictor reflexes [7]. Hydrostatic pressure and osmotic pressure plays a major role in the capillary fluid exchange. The imbalance between these two pressure leads to edema [18]. In the present study, we compared the plasma proteins between AIPE group and ATNE group, but there was no significance difference seen.

The antihypertensive effect of amlodipine is related to its mechanism of action. Amlodipine blocks the L- type calcium channels and relaxes the vascular smooth muscles. In contrast to arterioles, venules seem not to respond to L-type CCB [7]. The persistent Increase in catecholamine levels after chronic treatment with amlodipine enhances the release of $50 \%$ more catecholamine than the normal [19, 20]. In our study, we found significantly higher $24 \mathrm{~h}$. Urine VMA in AIPE group than ATNE group. VMA is ending metabolite of catecholamine [21]. The sympathetic stimulation or elevated plasma concentrations of catecholamine play a major role in edema [22].

Amlodipine inhibits the precapillary vasoconstriction through arteriolar dilatation [8]. However, amlodipine has less effect on venules; the increased catecholamine by chronic amlodipine therapy [19], which acts on adrenergic receptors on venules, which results in constriction of venules. So this produces the imbalance between the inflow and outflow between arterioles and venules. The difference in pressure gradient between arterioles and venules which possibly 
producing pedal edema. From this study, we can predict that catecholamine plays a major role in amlodipine-induced pedal edema.

The solution to resolving the amlodipine-induced pedal edema is to stop the monotherapy of amlodipine and start with combination therapy or switching to $\mathrm{L} / \mathrm{N}$ type of calcium channel blocker which will suppress catecholamine release from sympathetic nerves, and it helps in dilating both arterioles and venules, which may lead to the lesser incidence of edema.

\section{Limitations}

- The present study was an observational study. Hence, it was difficult to avoid or assess bias, and the conclusions are not always easily applicable across a generalize population.

- A urine sample $(24$ h.) collected for VMA test. We did not monitor the outpatient's food habits, water, and salt intake; it may interfere the VMA level.

- The few study patients travelled to our hospital from long distance with upright posture; it may vary the pedal edema size.

\section{CONCLUSION}

In essential hypertensive patients with AIPE group presented with a higher VMA level than the ATNE group. The elevated catecholamine's possibly the causative factor for AIPE.

\section{ACKNOWLEDGMENT}

The authors thank all the faculties of the Department of Medicine at Kasturba Hospital, Manipal University, Manipal, for providing study samples and Mrs. Vrinda, nursing staff, who helped us in blood samples collection. All authors read and approved the final version of the manuscript.

\section{CONFLICT OF INTERESTS}

Declared none

\section{REFERENCES}

1. Tripathi P, Venkataraman R, Bp SK, Kumaraswamy M, Singh R, Pandey $\mathrm{M}$, et al. Lifestyle factors: an alarm towards hypertension. Asian J Pharm Clin Res 2013;6:185-8.

2. Anchala R, Kannuri NK, Pant H, Khan H, Franco OH, Di Angelantonio E, et al. hypertension in India: a systematic review and meta-analysis of prevalence, awareness, and control of hypertension. J Hypertens 2014;32:1170-7.

3. Stafylas PC, Sarafidis PA. Carvedilol in hypertension treatment. Vasc Health Risk Manage 2008;4:23-30.

4. Schiffrin EL, Lipman ML, Mann JF. Chronic kidney disease: effects on the cardiovascular system. Circulation 2007;116:85-97.

5. Owen AJ, Reid CM. Cardio classics revisited: focus on the role of amlodipine. Integr Blood Pressure Control 2012;5:1-7.

6. James PA, Oparil S, Carter BL, Cushman WC, DennisonHimmelfarb C, Handler J, et al. Evidence-based guideline for the management of high blood pressure in adults: a report from the panel members appointed to the Eighth Joint National Committee (JNC 8). JAMA 2014;311:507-20.
7. Adake P, Somashekar HS, Mohammed Rafeeq PK, Umar D, Basheer B, Baroudi K. Comparison of amlodipine with cilnidipine on antihypertensive efficacy and incidence of pedal edema in mild to moderate hypertensive individuals: a prospective study. J Adv Pharm Technol Res 2015;6:81-5.

8. Shetty R, Vivek G, Naha K, Tumkur A, Raj A, Bairy KL. Excellent tolerance to cilnidipine in hypertensives with amlodipineinduced edema. North Am J Med Sci 2013;5:47-50.

9. Weir MR. The incidence of pedal edema formation with dihydropyridine calcium channel blockers: issues and practical significance. J Clin Hypertens 2002;5:330-5.

10. Sica DA. Calcium channel blocker-related peripheral edema: can it be resolved? J Clin Hypertens 2003;5:291-5.

11. Weir MR. The incidence of pedal edema formation with dihydropyridine calcium channel blockers: issues and practical significance. J Clin Hypertens 2003;5:330-5.

12. Oike M, Inoue $Y$, Kitamura K, Kuriyama H. Dual action of FRC8653, a novel dihydropyridine derivative, on the $\mathrm{Ba} 2+$ current recorded from the rabbit basilar artery. Circ Res 1990;67:993-1006.

13. Maheshwari P. A comparative study to evaluate the efficacy of amlodipine with bisoprolol in hypertensive patients. Asian J Pharm Clin Res 2014;7:98-102.

14. Jonas BS, Franks P, Ingram DD. Are symptoms of anxiety and depression risk factors for hypertension? Longitudinal evidence from the national health and nutrition examination survey i epidemiologic follow-up study. Arch Family Med 1997;6:43-9.

15. Pan Y, Cai W, Cheng Q, Dong W, An T, Yan J. Association between anxiety and hypertension: a systematic review and meta-analysis of epidemiological studies. Neuropsychiatr Dis Treat 2014;11:1121-30.

16. Player MS, Peterson LE. Anxiety disorders, hypertension, and cardiovascular risk: a review. Int J Psychiatry Med 2011;41:365-77.

17. Pedrinelli R, Dell'Omo G, Mariani M. Calcium channel blockers, postural vasoconstriction and dependent edema in essential hypertension. J Hum Hypertens 2001;15:455-61.

18. Messing M, Van Essen H, Smith TL, Smits JF, S Boudier HA. Microvascular actions of calcium channel antagonists. Eur J Pharmacol 1991;198:189-95.

19. De Champlain J, Karas M, Nguven P. Different effect of nifedipine and amlodipine on circulatory catecholamine level in essential hypertensive patients. J Hypertens 1998;16:1357-69.

20. Milovanovic B, Trifunovic D, Milicevic N, Vasic K, Krotin M. The significance of amlodipine on autonomic nervous system adjustment (ANSA Method): a new approach in the treatment of hypertension. Srp Arh Celok Lek 2009;137:371-8.

21. Graeme Eisenhofer, Irwin J Kopin, David S Goldstein. catecholamine metabolism: a contemporary view with implications for physiology and medicine. Pharmacol Rev 2004;56:331-49.

22. Rassler B. Contribution of $\alpha$-and $\beta$-adrenergic mechanisms to the development of pulmonary edema. Scientifica 2012:1-11. Doi:10.6064/2012/829504.

\section{How to cite this article}

- Kiran Shetty, Ranjan Shetty K, Pragna Rao, Vivek G, Naveenchandra GS, Abdul Razak, Deepak, Amruth Kiran Evaluation of causative factors in amlodipine-induced pedal edema. Int J Pharm Pharm Sci 2016;8(11):303-306. 